\title{
A FILTERBANK STRUCTURE FOR VOICE-BAND PCM CHANNEL PRE-EQUALIZATION
}

\author{
Nader Sheikholeslami Alagha Peter Kabal \\ Department of Electrical \& Computer Engineering \\ McGill University, Montreal, Canada
}

\begin{abstract}
A non-maximally decimated filterbank structure for preequalizing channels with Inter-Symbol Interference (ISI) is investigated. The impulse response of the channel is assumed to be known at the transmitter. Compared with the classical Tomlinson-Harashima Precoding technique, the proposed pre-equalizer compensates for the channel without increasing the number of the received signal levels (channel alphabet). The proposed technique does not require the channel to be minimum-phase. The filterbank structure adds redundancy to the input signal to compensate for the channel ISI while keeping the transmitted power bounded.

The proposed pre-equalization is particularly useful for data transmission over voice-band PCM channels. The upstream PCM channel is bandlimited, causing severe ISI at the output of the front-end receiver filter. By using the preequalizer at the transmitter, channel ISI can be mitigated.
\end{abstract}

\section{INTRODUCTION: PCM MODEMs}

In many parts of the world, the Public Switching Telephone Network (PSTN) has evolved towards a digital communication network. The interconnections among central offices are fully digital. The only remaining analog links are the analog subscriber loops connecting subscriber premises to the serving central office.

At the central office, the received signal from an analog subscriber loop is converted to a stream of binary digits. The typical encoding scheme used at the central offices is Pulse Code Modulation (PCM) [1]. A PCM encoder performs three operations on the input signal: filtering, sampling and quantization. The front end filter is used to limit the bandwidth of the input signal to less than one half of the sampling frequency. Based on international PCM standards for voice telephony [2], sampling is done at 8000 samples per second. A non-uniform quantizer ( $\mu$-law or Alaw) maps the amplitude of each sample to an 8-bit binary codeword [1]. The output of a PCM encoder is a $64 \mathrm{~kb} / \mathrm{s}$ DS0 bit stream corresponding to each voice channel.

A conventional voice-band modem is designed to transmit data between two users both connected to analog subscriber lines. These modems view the voice channel as an analog medium, treating the A/D conversion error in PCM

Web page: http://WWW.TSP.ECE.McGill.CA. encoding process as an additive noise. The main source of signal distortion in this channel is quantization distortion. Traditionally, the quantization error is modeled as additive white Gaussian noise (AWGN). For a typical voice channel $W$ is $3-3.5 \mathrm{kHz}$. The predominant noise is the quantization error introduced by PCM encoding. For non-uniform 8-bit quantizer, the signal-to-quantization-noise-ratio (SQNR) is typically in the range of 33-39 $\mathrm{dB}$ giving a channel capacity of $33-45 \mathrm{~kb} / \mathrm{s}$. In the absence of quantization error, the capacity of the PCM voice channel is limited by the bandwidth of the channel and the resolution of the PCM quantizer. The maximum rate of independent symbols over a bandlimited channel is twice the bandwidth of the channel. Since each symbol is represented by 8 bits the maximum information rate is $16 \mathrm{~W}$ bit/s. For typical values of $W$, the maximum information rate is $48-56 \mathrm{~kb} / \mathrm{s}$.

The idea of modem design specifically for PCM voiceband channels were first reported in [3]. The central idea of this design is to avoid the effects of quantizer distortion by employing the quantizer decision levels as the channel symbol alphabet. In the up-stream direction, from the analog subscriber loop to the central office, the transmitted signal is adjusted so that the analog voltage sampled by the PCM encoder is equal to the desired level at the sampling instant. Therefore, the quantization distortion does not effect the transmitted symbols.

In the down-stream direction from the central office to the analog user, there is no quantization distortion. The signal in the down-stream direction can be treated as a base-band Pulse Amplitude Modulated (PAM) signal passing through a band-limited channel. Modems based on this approach are known as PCM modems [4].

Although the idea of using PCM modems for end-to-end analog subscribers is appealing in theory, it seems quite challenging to implement in practice. The connection between two end users consists of two separate links, with two different characteristics. Without side information from within the digital switched telephone network, it is difficult to solve problems such as synchronization, echo cancellation and channel equalization for the end-to-end connection between two analog subscriber loops.

The growing demand for Internet access has created a new trend of data connection on the telephone network. At one end, there is a residential computer user who connects to the telephone network through an analog subscriber loop. At the other end, there is an Internet service provider with direct digital access to the switched network 


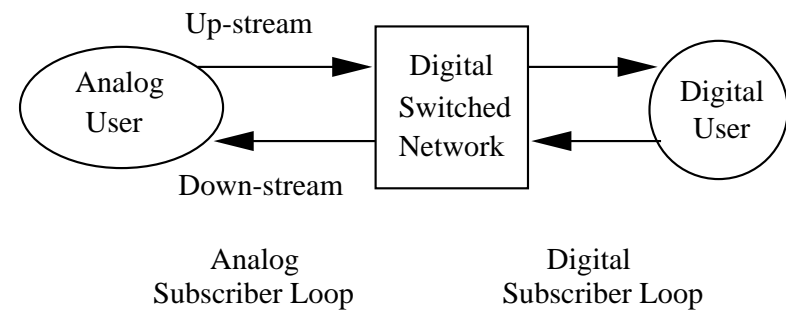

Fig. 1 Hybrid connection between Analog and digital subscriber loops

via a T1 link or ISDN (Fig. 1). In this configuration, many issues associated with PCM modem design become less complicated. The most recent ITU-T recommendation, the V.90 Standard [5], provides guidelines for designing PCM modems in down-stream direction to achieve throughput up to $56 \mathrm{~kb} / \mathrm{s}^{1}$.

Modem design for the up-stream PCM channel, connecting the end user to the central office, is a more challenging task. The challenge is primarily due to the quantization distortion at the PCM encoder and the bandlimited filtering at the receiver front end ${ }^{2}$. A conventional analog modem models the distortion caused by the PCM encoder as an additive noise. Unlike the analog modem, a PCM modem treats the PCM encoder as a front end receiver block which contains a band-pass filter, a sampler and a symbol detector (the quantizer). However, the choice of receiver analog filter, sampling rate, sample timing and the symbol detector are constrained by the network hardware. The anti-aliasing filter at the central office along with the subscriber loop filtering effectively limit the frequency band of the up-stream channel to the $300-3600 \mathrm{~Hz}$ range. While the channel bandwidth is restricted to about $3300 \mathrm{~Hz}$, the sampling frequency at the central office is fixed at 8000 $\mathrm{Hz}$. According to Nyquist sampling theorem, the maximum number of independent samples transmitted over this channel is limited to 6600 samples per second. In order to avoid ISI, the transmitter should compensate for the channel oversampling.

In Section 2 several techniques for channel preequalization are described. In Section 3 we propose a filterbank structure to compensate for the channel. Some examples and simulation results are presented in Section 4.

\section{CHANNEL PRE-EQUALIZATION}

If one assumes that the channel characteristics are known at the receiver as well as the transmitter (possibly via a feedback channel), one can perform the equalization at either end of the channel. By performing channel equalization at the transmitter, we can avoid noise enhancement, as

\footnotetext{
${ }^{1}$ In North America, due to some regulatory power constraints, this number is limited to $53 \mathrm{~kb} / \mathrm{s}$. The regulations are expected to change in the near future.

${ }^{2}$ In the V.90 Standard, the conventional analog data transmission based on V.34 Standard has been adopted in the up-stream direction [5]
}

in a linear equalizer at the receiver, and avoid error propagation, as in a decision feedback equalizer. However, a straightforward pre-filtering can increase the transmit signal power.

Let us assume that a voice-band PCM channel is characterized by a linear time-invariant FIR filter. Furthermore, we assume that the channel is causal and monic. This model can be characterized as:

$$
H(z)=1+\sum_{i=1}^{M-1} h(i) z^{-i}
$$

A perfect equalizer for the channel is a filter with $1 / H(z)$ transfer function. Due to spectral nulls, the inverse filter may not have a stable realization.

Tomlinson-Harashima precoding (TH-precoding) is a solution to pre-filtering without significantly increasing the peak and average transmitted power $[6,7]$. There is however, a serious drawback associated with TH-precoder; it increases the dynamic range of the received signal [8]. This increase in dynamic range can be particularly severe if the spectrum of the channel contains spectral nulls. If the original symbols are taken from a finite set, TH-precoder will increase the size of this set for the received signal. For the up-stream PCM channels, the number of receiver levels is limited by the number of levels of the non-uniform quantizer. To use a TH-precoder for the PCM channel we must reduce the number of channel input levels to avoid any overload error at the receiver front end. Due to these limitations, TH-precoder is not appropriate choice of precoding for the up-stream PCM channel. in

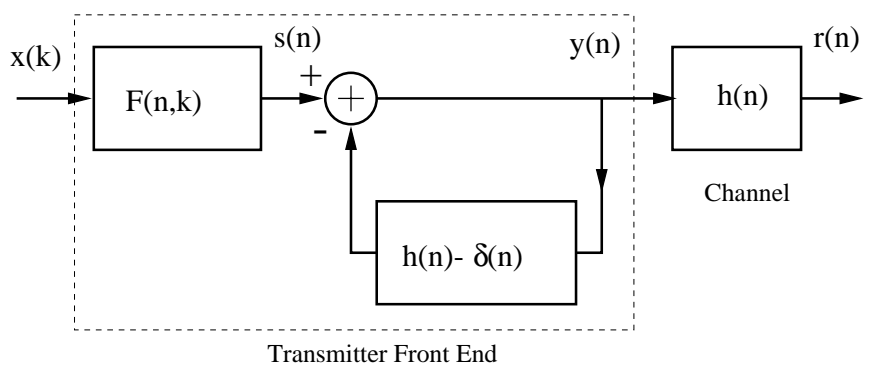

Fig. 2 Linear Time Varying Precoder

We previously proposed a linear time-varying filtering technique to equalize the channel at the transmitter [9]. Figure 2 shows the proposed structure. The inverse filter, $1 / H(z)$, is realized using a direct form structure with a feedback connection. $F(n, k)$ is a linear time-varying filter that extends a block of $(N-M)$ inputs to $N$ outputs where $M$ is the length of the channel impulse response. Added symbols are used to cancel the poles of the inverse filter $1 / H(z)$.

The general structure of the time-varying pre-equalizer can result in many different implementations. One structure was given in [9] where a simple on-off switch resets the filter feedback loop to the zero state (see Fig. 3).

An alternative approach for PCM channel equalization was reported in [4] where the channel is assumed unknown 


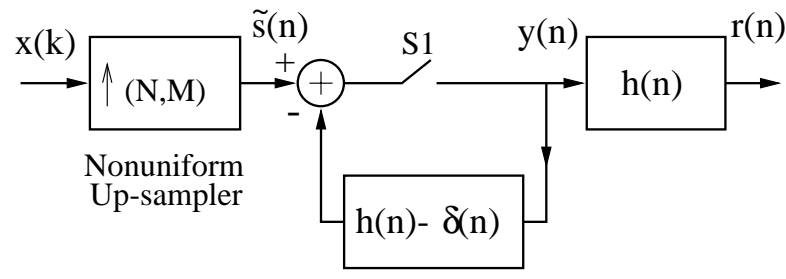

Fig. 3 A simple implementation of Time Varying Precoder

at the transmitter. For the case of ideal bandlimited channel, a set of signals with a regular pattern of zero crossings was derived.

A precoding/equalization technique for ISI channel was proposed in [10], where the precoder non-uniformly upsamples the symbols before transmission. At the receiver a filterbank is in place to equalize the channel. this scheme requires an equalizer at the receiver which is not applicable for the up-stream PCM channel.

\section{FILTERBANK PRE-EQUALIZERS}

Assuming that the channel is known at the transmitter as an FIR filter, we consider a non-maximally decimated filterbank [11] to accommodate redundant symbols along with the information carrying symbols in the transmitted signal. Since not all the received symbols carry useful information, the system can tolerate ISI at certain sampling instances at the receiver. The "don't care" samples are used to avoid ISI at the other sampling instances. Figure (4) shows the structure of the filterbank.

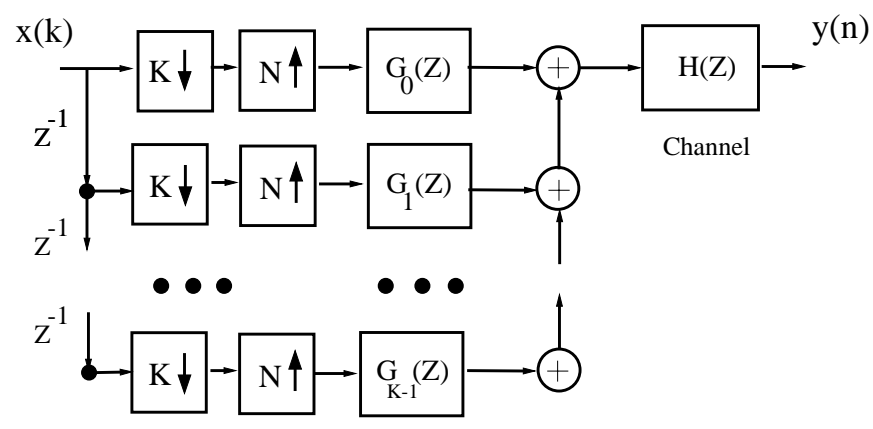

Pre-Equalizer Filterbank

Fig. 4 A simple implementation of Time Varying Precoder

Note that the decimation factor $K$ is less than upsampling rate $N$. Each branch of the filterbank contains an linear time-invariant filter but the overall filterbank is periodically time varying system. We will show that these filters can be chosen to be FIR, even though the inverse filter $1 / H(z)$ is an IIR filter. Filters are designed such that ISI for each branch and inter-channel Interference (ICI) between different branches are avoided. Let us consider the condition for the first filter:

$$
g_{0}(n) * h(n)= \begin{cases}1 & n=0 \\ 0 & n=N m+l, l<K\end{cases}
$$

where $m$ and $l$ are non-negative integers. In the $z$-domain the condition for all the filters can be written as:

$$
G_{k}(z) H(z)=z^{-k}+\sum_{i=0}^{\infty} \sum_{j=K}^{N-1} d(i, j) z^{(-N i-j)}
$$

where $0 \leq k \leq K-1$ is the branch index. The coefficients $d(i, j)$ are at the sampling instances that do not contain information. These coefficients are used to provide appropriate spectral shaping for $G_{k}(z)$ :

$$
G_{k}(z)=\frac{z^{-k}+\sum_{i=0}^{L} \sum_{j=K}^{N-1} d(i, j) z^{(-N i-j)}}{H(z)}
$$

Coefficients $d(i, j)$ can be chosen such that the poles of $G_{k}(z)$ are all cancelled and the filters become FIR . Since $H(z)$ has finite number of roots (say $M$ ), the number of coefficients required for pole-zero cancellation is also finite.

As an example, consider a simple channel $H(z)=1+z^{-1}$. For a filterbank structure with $N=4$ and $K=3$, the filters are determined as:

$$
\begin{aligned}
& G_{0}(z)=\frac{1-z^{-3}}{1+z^{-1}}=1+z^{-1}+z^{-2} \\
& G_{1}(z)=\frac{z^{-1}-z^{-3}}{1+z^{-1}}=z^{-1}+z^{-2} \\
& G_{2}(z)=\frac{z^{-2}-z^{-3}}{1+z^{-1}}=z^{-2}
\end{aligned}
$$

As shown in this example, to eliminate the tail of the inverse channel impulse response $h_{i n v}(n)$, the impulse response is delayed, scaled and subtracted from the original impulse. For example $g_{k}(n)$ can be written as:

$$
g_{k}(n)=h_{i n v}(n-k)+\sum_{i=0}^{L} \sum_{j=K}^{N-1} d(i, j) h_{i n v}(n-N i-j)
$$

The value of $g_{k}(n)$ is forced to zero after a finite number of samples. In the context of FIR filter implementation, this technique has been used to implement an FIR filter as a tail-cancelling IIR filter [12].

Since the filterbank structure contains only FIR filters, the transmitter power is always bounded. However, depending on the channel filter and the choice of up-sampling and down-sampling factors in the filterbank, the total filter gain can be larger than one. 


\section{SIMULATION RESULTS}

Consider a channel filter with spectral nulls:

$H(z)=\left(1-z^{-1}\right)\left(1+z^{-1}\right)\left(1-e^{j 0.8 \pi} z^{-1}\right)\left(1-e^{-j 0.8 \pi} z^{-1}\right)$

We design a pre-equalizer for this channel using the filterbank structure. Several up-sampling and down-sampling factors for the filterbank are considered. As an obvious choice, we would like to gain the maximum throughput from the channel. The decimation ratio $K / N$ is directly related to the throughput. As a measure of transmitted power, we use the average magnitude squared of the filter coefficients. Table 1 shows the simulation results. It is evident that increasing the decimation rate does not always lead to increasing of the average transmitted power.

The last row of the table also shows that for the given up-sampling/downsampling factors, there are no finite solutions for the FIR filter (degenerate case).

Table 1 Comparison of decimation rates and the average filter gain

\begin{tabular}{cccc}
\hline \hline$K$ & $N$ & Decimation Rate & $\frac{1}{K} \sum_{k=0}^{K-1} \sum_{n=0}^{N_{k}}\left|g_{k}(n)\right|^{2}$ \\
\hline 3 & 8 & 0.375 & 5.03 \\
4 & 8 & 0.500 & 8.10 \\
5 & 8 & 0.625 & 55.98 \\
6 & 8 & 0.750 & 44.57 \\
7 & 8 & 0.875 & $>>1$ \\
\hline
\end{tabular}

\section{CONCLUDING REMARKS}

We have investigated a new pre-equalizer structure for the up-stream PCM channel. The pre-equalizer is implemented as FIR filters with in a filterbank structure. Since the filterbank is not maximally decimated, the output of the pre-equalizer contains redundancy. The FIR filters use the redundant symbols to avoid ISI at sampling instances that contain information. The peak and average transmitted power are function of the up-sampling and down-sampling factors used in the filterbank.

\section{References}

[1] W. D. Reeve, Subscriber Loop Signaling and Transmission Handbook, Digital. IEEE Press, 1995.

[2] Recommendation G.711, "Pulse code modulation (PCM) of voice frequencies," CCITT Series G Recommendations, 1988.

[3] I. Kalet and J. E. Mazo and B. R. Saltzberg, "The capacity of PCM voiceband channels," Proc. IEEE Int. Conf. Communications, pp. 507-511, 1993.

[4] E. Ayanoglu, N. R. Dagdeviren, G. D. Golden, and J. E. Mazo, "An equalizer design technique for the PCM modem: A new modem for the digital public switched network," IEEE Trans. Communications, vol. 46, pp. 763-774, June 1998.

[5] ITU-T Recommendation V.90, "A digital modem and analogue modem pair for ue on the public switched telephone network (PSTN) at data signalling rates of up to $56000 \mathrm{bit} / \mathrm{s}$ downstream and up to 336000 bit.s upstream," 1998.

[6] M. Tomlinson, "New automatic equaliser employing modulo arithmetic," Electronics Letters, vol. 7, pp. 138-139, March 1971.

[7] E. A. Lee and D. G. Messerschmitt, Digital Communication. KAP, 1994.

[8] R. F. H. Fischer and J. B. Huber, "Comparison of precoding schemes for digital subscriber lines," IEEECOM, vol. 45, pp. 334-343, March 1997.

[9] Nader Sheikholeslami and Peter Kabal, "Linear time varying precoder applied to ISI channel," IEEE Pacific Rim Conf. Commun., Computers and Sig. Processing, pp. 36-39, 1997.

[10] X.-G. Xia, "New precoding for intersymbol interference cancellation using non-maximally decimated multirate filterbanks with ideal fir equalizers," IEEE Trans. Signal Processing, vol. 45, pp. 2431-2441, October 1997.

[11] P. Vaidyanathan, Multirate Systems and Filter banks. Prentice-Hall, 1993.

[12] A. Wang and J. Smith, "On fast FIR filters implemented as tale-canceling IIR filters," IEEE Trans. Signal Processing, vol. 45, pp. 1415-1427, June 1997. 